\title{
A numerical study of heat transfer and fluid flow over an in-line tube bank
}

\author{
Z. S. Abdel-Rehim \\ Mechanical Engineering Department, National Research Center, Egypt
}

\begin{abstract}
Heat transfer and fluid flow over an in-line tube bank using a computational fluid dynamic (CFD) prediction is presented in this study. The study considers steady laminar two-dimensional incompressible flow over in-line tube bundles used in heat exchanger applications. The effect of various independent parameters, such as Reynolds number $(\mathrm{Re})$, length aspect ratio $(\mathrm{L} / \mathrm{D}=2.5,2,1.5,1.0,0.5$ and 0.0$)$, and fluid flow on the pressure drop and heat transfer is studied. A computer code (CFD-Fluent) is used, generally, to predict the heat transfer and pressure drop characteristics of flows around or through rigid complex geometry, namely domains whose boundaries do not coincide with coordinate lines of a Cartesian or any simple coordinate system.

A comparison is made between the present CFD predictions and reference measurements (Iwaki, et al. PIV Measurement of Vertical Cross Flow Structure over Tube Bundles, Experiments in Fluids, 2004) for the velocity vector as fluid flow over a cylinder in cross flow for the selected values of Reynolds numbers $\mathrm{Re}_{\mathrm{D}}$. Therefore, the comparison is made between the present CFD results and Martin empirical correlation of an in-line circular tube for the variation of $\mathrm{Nu}$ with $\mathrm{Re}_{\mathrm{D}}$. A reasonable agreement is observed. The results of the present study provide sufficient confidence in using empirical correlations to undertake initial sizing of tube bank heat exchanger design, with thermo-fluid performance subsequently predicted by CFD analysis for specific requirement applications.

Keywords: CFD prediction, fluid flow, in-line arrangement, laminar flow, length aspect ratio, tube bank.
\end{abstract}

\section{Introduction}

Heat transfer enhancement is the process of improving the performance of a heat transfer system by increasing the heat transfer coefficient. Flat tubes have not 
been investigated to the same extent, although they play an important role in many technical applications such as, modern heat exchangers, automotive radiators, air conditioning evaporators and condensers (Webb [1]). Finite volume techniques are increasingly being used for computing incompressible flow in arbitrary geometries because of the recent developments in grid generation methods. An Introduction to Computational Fluid Dynamics the Finite Volume Method (Versteeg and Malalasekera [2]). Grid generation within two- and threedimensional spaces is considerably more complicated than one-dimensional grid generation (Hoffman [3]). Most studies were concerned with the flow over a circular cylinder (Williamson 1996 and Zdrakovich [4]). Extensive numerical based studies have been undertaken to investigate the fluid flow and heat transfer in tube banks (Fujii et al. [5]).

The main objective of this work is to develop a computer code to predict the heat transfer and pressure drop characteristics of flow (for simplicity) through one vertical row of in-line alignment of tube bank. The present study considers steady laminar two-dimensional incompressible flow past tube found in heat exchanger applications. The main purpose of this work is to study the effect of different length aspect ratios $(\mathrm{L} / \mathrm{D}=2.5,2,1.5,1.0,0.5$ and 0.0$)$ and Reynolds numbers of the fluid on pressure drop and heat transfer. The results present the grid, residual, static pressure, static temperature and velocity vector for the selected cases at the same conditions, assumptions and the values of Reynolds numbers. A comparison is made between the present CFD predictions and reference measurements (Iwaki et al. [6]) for the velocity vector and magnitude as fluid flow over a cylinder in cross flow for the selected values of Reynolds numbers $\mathrm{Re}_{\mathrm{D}}$. Also, the comparison is made between the present CFD results and Martin empirical correlation of in-line circular tube for the variation of $\mathrm{Nu}$ with $\mathrm{Re}_{\mathrm{D}}$.

\section{Governing equations and solution method}

The differential equations governing the conservation of mass, momentum, and energy can be cast into a general form as:

$$
\frac{\partial(\rho \phi)}{\partial t}+\nabla \cdot \vec{J}=S
$$

where $\mathrm{t}$ is time, second

where

$$
\begin{gathered}
\vec{J}=\rho \vec{V} \phi-\Gamma \nabla \phi \\
\vec{J}=\left(\rho U \phi-\Gamma \frac{\partial \phi}{\partial X}\right) e_{x}+\left(\rho V \phi-\Gamma \frac{\partial \phi}{\partial y}\right) e_{y}
\end{gathered}
$$

In these equations, $\boldsymbol{\varphi}$ is a general dependent variable $(\varphi=U, V, p$ and $T), \rho$ is the mass density kg.m ${ }^{-3}, \Gamma$ is the effective diffusion coefficient, $\vec{V}$ is the velocity vector, and $S$ designates the volumetric source (or sink) of $\varphi$. In Eqn. (2) $\vec{J}$ corresponds to the total flux $\mathrm{W} / \mathrm{m}^{2}$ of $\varphi$, i.e., it takes account of both the 
convective and diffusive fluxes. For convenience, expressions in the Cartesian vector notation for steady-state incompressible flow are given below.

$$
\text { Continuity: } \frac{\partial U}{\partial x}+\frac{\partial V}{\partial y}=0.0
$$

where $U, V$ are Cartesian components of fluid velocity, $\mathrm{m} \cdot \mathrm{s}^{-1}$ and $x, y$ are Cartesian coordinates.

$$
\text { Momentum: } \rho(\vec{V} . \nabla) \vec{V}=-\nabla P+\mu \nabla^{2} \vec{V}
$$

where $P$ is pressure drop, pa and $\mu$ is dynamic viscosity, N.s.m ${ }^{-2}$.

Momentum Equation in Two Directions:

$$
\begin{aligned}
& \frac{\partial(U U)}{\partial x}+\frac{\partial(U V)}{\partial y}=-\frac{\partial P}{\partial x}+\frac{1}{\mathrm{Re}}\left(\frac{\partial^{2} U}{\partial^{2} x}+\frac{\partial^{2} V}{\partial^{2} y}\right) \\
& \frac{\partial(U V)}{\partial x}+\frac{\partial(V V)}{\partial y}=-\frac{\partial P}{\partial y}+\frac{1}{\mathrm{Re}}\left(\frac{\partial^{2} U}{\partial^{2} x}+\frac{\partial^{2} V}{\partial^{2} y}\right)
\end{aligned}
$$

where $\mathrm{Re}$ is Reynolds number $\left(=\rho \mathrm{VD}_{\mathrm{h}} / \mu\right)$

$$
\text { Energy: } \quad \rho C_{P}(\vec{V} \cdot \nabla) T=k \nabla^{2} T
$$

where $C_{p}$ is specific heat, $\mathrm{J} \cdot \mathrm{kg}^{-1} \cdot \mathrm{K}^{-1}, \mathrm{~T}$ is temperature, $\mathrm{K}$ and $\mathrm{k}$ is thermal conductivity, W. $\mathrm{m}^{-1} \cdot \mathrm{K}^{-1}$

$$
\frac{\partial(U T)}{\partial x}+\frac{\partial(V T)}{\partial y}=\frac{1}{\operatorname{Re} \operatorname{Pr}}\left(\frac{\partial^{2} U}{\partial^{2} x}+\frac{\partial^{2} V}{\partial^{2} y}\right)
$$

where Pr is Prandtl number $\left(=\mu C_{p} / \mathrm{k}\right)=0.7$ (air)

Wall Boundary Conditions, the most common boundary condition that comes across in a confined fluid flow problem is the wall boundary condition. The noslip condition, valid for viscous flows, can be enforced for the velocities. Consequently, the velocity of the fluid at the wall must be equal to the velocity of the wall (i.e., zero in most of the cases).

Eqns. (4)-(9) above are referred to as the Navier-Stokes equation for the general constant property incompressible flow of a Newtonian fluid. The Navier-Stokes equation in the above form constitute a system of two equations (in two-dimensional flow) with two unknowns, $\mathrm{P}$ and $\vec{V}$.

Solution method, the in-line, implicit solver is used to solve the governing differential equations for the conservation of the mass, momentum and energy equations.

\section{Mathematical assumptions}

- The flow is two-dimensional, steady, laminar and incompressible. 
- The thickness of the tube is neglected.

- The thermo-physical properties of the fluid do not vary with temperature.

- The viscous heating is neglected.

\section{Control volume formulations}

The first step to derive the discretized equation from the general conservation equation is to divide the computational domain into a set of non-overlapping control volumes then the conservation equations are expressed in an integral form in each of these control volumes. The advantage of this control volume approach is to preserve the conservation property, which is important for engineering applications in which the overall balance of mass, momentum, and energy are usually of major significance. The formulation presented here is identical to the formulation proposed by Karki [7]. The computational domain, in two-dimension, is divided into quadrilateral volumes by using any of the grid generation techniques. A Numerical study of heat and momentum transfer over flat tubes is presented by Haitham and Bahaidarah [8].

\section{Structured grids}

Two-dimensional, steady state form of the generalized Eqn. (1) is discretized as:

$$
\nabla \cdot \vec{J}=S
$$

This equation will be discretized in terms of fluxes. Then, the general transport equation for property $\varphi$ is derived by using a proper profile assumption. The control volume integration, which represents the main step of the finite volume technique, yields the following form:

$$
\int_{A} \vec{J} \cdot e^{X} d S=\int_{V} S d V
$$

where $V$ is the volume of a curvilinear element with a fixed boundary and $e^{x}$ is a general unit normal vector in one cell face.

\section{Geometry, grid distribution and boundary conditions for in-line configuration}

Figure 1 shows the grid configuration, initial, boundary conditions and control volume of the present model. In this study, (for simplicity), one vertical row of in-line tube bank and four tubes are included in the computational domain.

The computational domain was divided into three individual regions as; entry region, tube module and exit region. A uniform orthogonal grid was used for both entry and exit regions.

Where $\mathrm{D}$ is diameter, $\mathrm{m}, \mathrm{H}$ is height of control volume, $\mathrm{m}$ and $\mathrm{L}$ is length, $\mathrm{m}$. The surfaces BC and FG are the top wall of the lower tube and the bottom wall of the upper tube, respectively. A no-slip boundary condition was assigned for 
these two surfaces. The lines AB, CD, EF, and GH are lines of symmetry where no flow crosses these boundaries and the normal component of velocities and the normal gradient of the parallel component of velocity are set to zero. Finally, the lines $\mathrm{AH}$ and $\mathrm{DE}$ are recognized as the module inlet and module outlet, respectively.

Figure 2 shows the model of length aspect ratio.

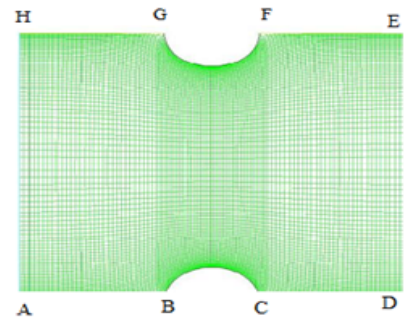

BC: surface of top wall of the lower tube FG: surface of bottom wall of the upper tube

$\mathrm{AB}, \mathrm{CD}, \mathrm{EF}$ and $\mathrm{GH}$ : lines of symmetry

$\mathrm{AH}$ : line of module inlet

DE: line of module outlet

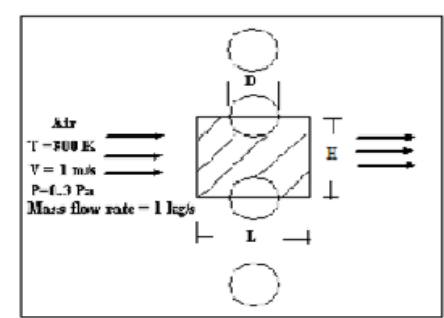

Vertical one row, control volume and initial conditions

Figure 1: Grid configuration, initial and boundary conditions and control volume of the present model.

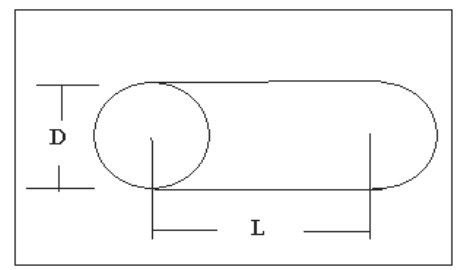

Figure 2: $\quad$ Model of length aspect ratio.

\section{Correlations}

Martin [9] developed a comprehensive convective heat transfer correlations for in-line tube banks, in terms of Lévêque number, Lq (=0.5 $\left.\mathrm{X}_{\mathrm{T}} \mathrm{H}_{\mathrm{g}} \mathrm{pr} \mathrm{D}_{\mathrm{h}} / \mathrm{L}\right)$, where with Nusselt number as:

$$
N u=0.404 L q^{\frac{1}{3}}\left(\frac{\operatorname{Re}_{D}+1}{\operatorname{Re}_{D}+1000}\right)^{0.1}
$$

Although the correlations have been developed for the predication of the local $\mathrm{Nu}\left(=\mathrm{h} \mathrm{D}_{\mathrm{h}} / \mathrm{k}\right)$, the importance lies in the overall average heat transfer coefficient. The empirical correlation proposed by Hilpert [10] for the overall $\mathrm{Nu}$ is widely used: 


$$
\bar{N} u_{D}=\frac{\bar{h} D}{k}=C \cdot \operatorname{Re}_{D}{ }^{m} \cdot \operatorname{Pr}^{\frac{1}{3}}
$$

where $C$ and $m$ are the model constants. An alternative correlation for convective heat transfer from cylinders of low aspect ratio in the limit of the same form was proposed by Zhukauskas [11].

\section{Results and discussions}

The main purpose of this work is to study the effect of Reynolds number, aspect ratio $(\mathrm{L} / \mathrm{D}=2.5,2,1.5,1,0.5$ and 0 , i.e. from flat tube to circle tube), and air fluid flow over of the in-line tube bank on pressure drop and heat transfer. Figure 3 shows the residual solution for the selected length aspect ratio cases - in-line alignment at $\mathrm{Re}_{\mathrm{D}}=200$.

$\mathrm{L} / \mathrm{D}=2.5$

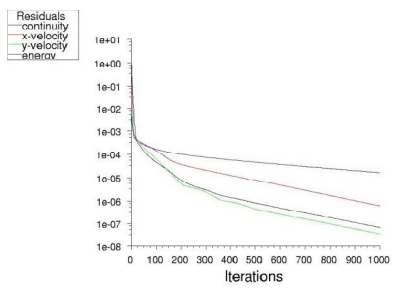

$\mathrm{L} / \mathrm{D}=1$

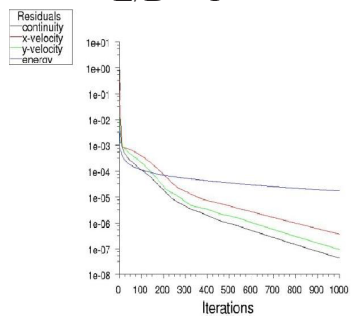

Iterations

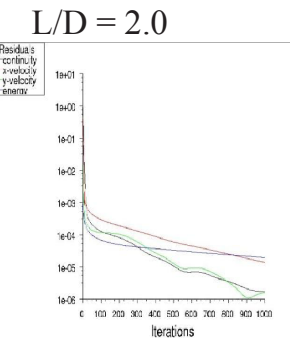

$\mathrm{L} / \mathrm{D}=0.5$

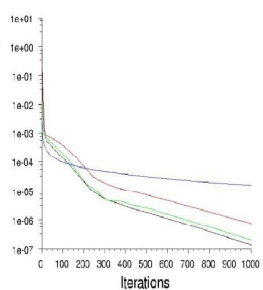

$\mathrm{L} / \mathrm{D}=1.5$

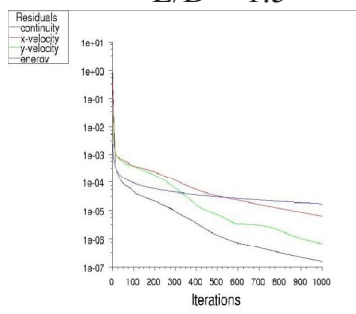

$$
\mathrm{L} / \mathrm{D}=0
$$

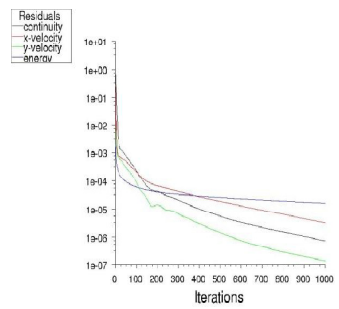

Figure 3: Residual solution for the selected length aspect ratio cases - in-line alignment at $\operatorname{Re}_{\mathrm{D}}=200$.

The contour of static pressure for the selected length aspect ratio cases - inline alignment at $\mathrm{Re}=200$ is presented in fig. 4 .

$$
\mathrm{L} / \mathrm{D}=2.5 \quad \mathrm{~L} / \mathrm{D}=2 \quad \mathrm{~L} / \mathrm{D}=1.5
$$

As the length aspect ratio increases the normalized pressure drop decreases. The contour of static temperature for the selected length aspect ratio cases - inline alignment at $\mathrm{Re}=200$ is given in fig. 5 . 


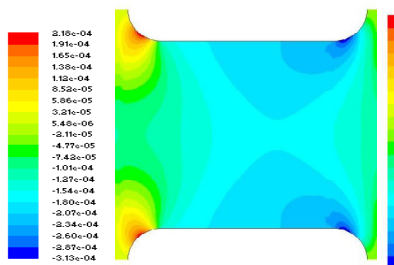

$\mathrm{L} / \mathrm{D}=1$
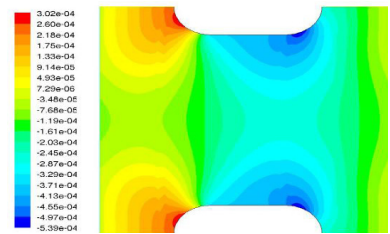
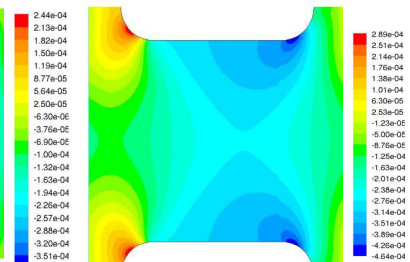

$\mathrm{L} / \mathrm{D}=0.5$

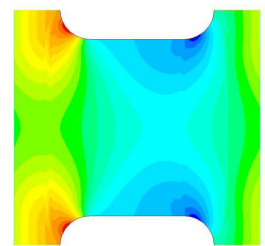

$\mathrm{L} / \mathrm{D}=0$
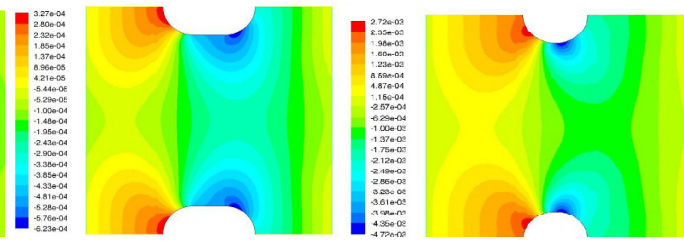

Figure 4: $\quad$ Contour of static pressure for the selected length aspect ratio cases - in-line alignment at $\operatorname{Re}=200$.

$\mathrm{L} / \mathrm{D}=2.5$
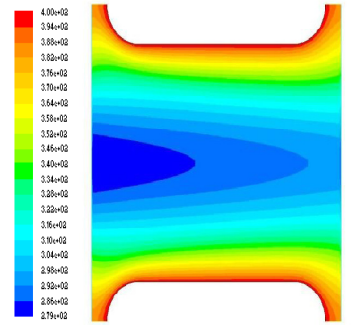

$\mathrm{L} / \mathrm{D}=1$

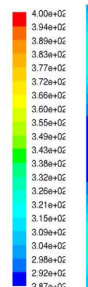

$\mathrm{L} / \mathrm{D}=2$
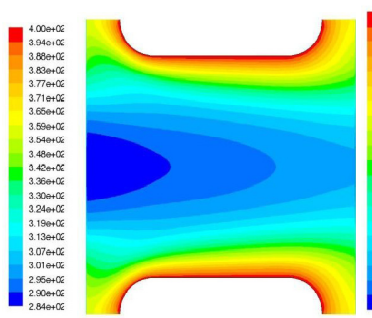

$\mathrm{L} / \mathrm{D}=.5$
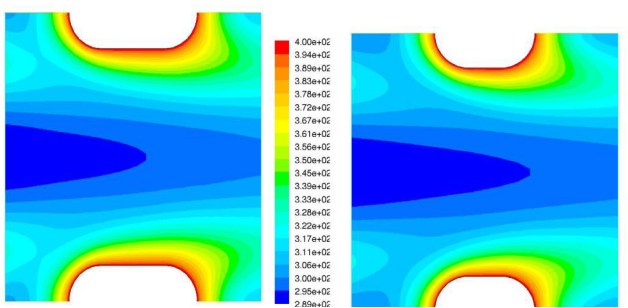

$\mathrm{L} / \mathrm{D}=1.5$

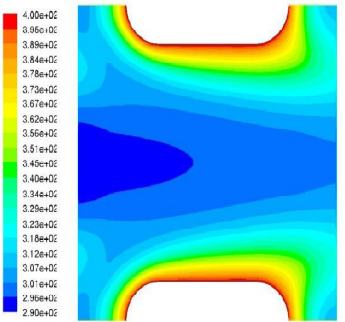

$\mathrm{L} / \mathrm{D}=0$
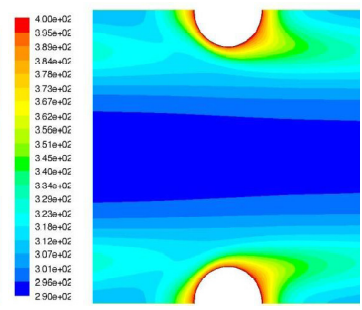

Figure 5: Contour of static temperature for the selected length aspect ratio cases-in-line alignment at $\mathrm{Re}=200$.

The results presented in this figure serve as excellent benchmark data for assessing CFD predictive accuracy for the selected aspect ratio cases. This behaviour is a function of the ratio of the pressure drop to velocity squared terms in the calculation of the temperature distribution. The contour of velocity vector for the selected length aspect ratio cases - in-line alignment at $\mathrm{Re}=200$ is depicted in fig. 6 . 
$\mathrm{L} / \mathrm{D}=2.5$
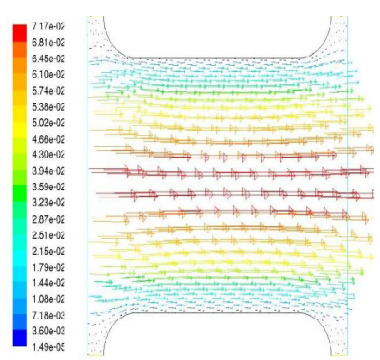

$\mathrm{L} / \mathrm{D}=1$
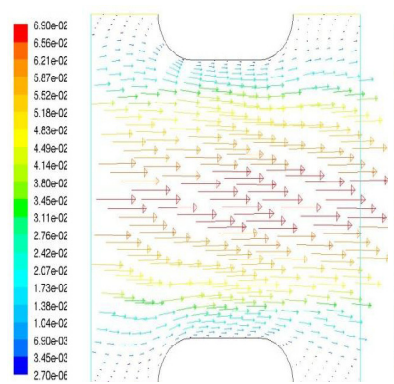

$\mathrm{L} / \mathrm{D}=2$
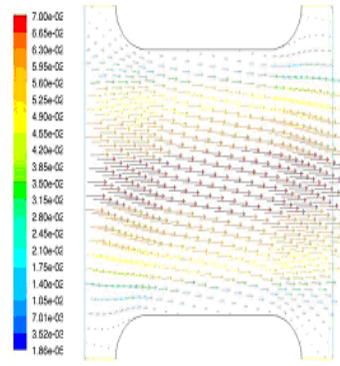

$\mathrm{L} / \mathrm{D}=0.5$
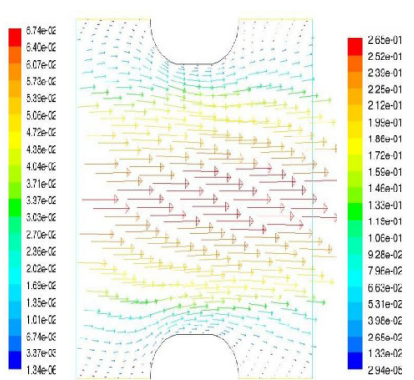

$\mathrm{L} / \mathrm{D}=1.5$

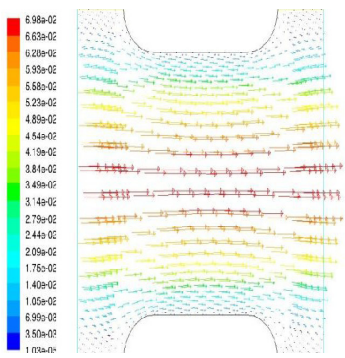

$\mathrm{L} / \mathrm{D}=0$

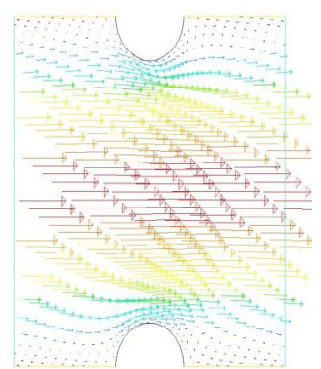

Figure 6: Contour of velocity vector for the selected length aspect ratio cases in-line alignment at $\mathrm{Re}=200$.

The overall performance of an in-line configuration with a lower length ratio $(\mathrm{L} / \mathrm{D}=0.0$, circle $)$ is preferable since it provides higher heat transfer rate. The heat transfer enhancement is obtained which indicates that circular tube out performs flat tube. The present results confirm the ability and potential of CFD analysis as a design tool, to provide detailed insight into complex heat transfer that would otherwise be difficult to characterize experimentally. As the Reynolds number increases the maximum velocity in the passage between the upper and lower tubes increases. Figures show that the colder fluid comes in contact with the hot tube surface as the Reynolds number increases. Accordingly, heat transfer increases with an increase in the Reynolds number.

A small change in the heat transfer rate can be predicted since the fluid has almost the same behavior when the length ratio is increased. Figure 7 shows the velocity profiles along the transverse direction of the module outlet and inlet wall for an in-line arrangement for higher length ratios, $L / D=2.5$ : at $R e=200$ and $\mathrm{Re}=400$. The velocity profile is nearly symmetric.

The velocity distribution is normalized by the value of the uniform inlet velocity $\mathrm{Uo}$ and given as a function of $\mathrm{Y} / \mathrm{H}$ position. The maximum velocity in the middle of $(Y / H)$ position the passage considerably decreases because of a larger flow area. Figure 8 shows the average Nusselt number as a function of Reynolds number for the present module for in-line configurations for $\operatorname{Pr}=0.71$. In general, the module average Nusselt number increases with an increase in Reynolds number. 


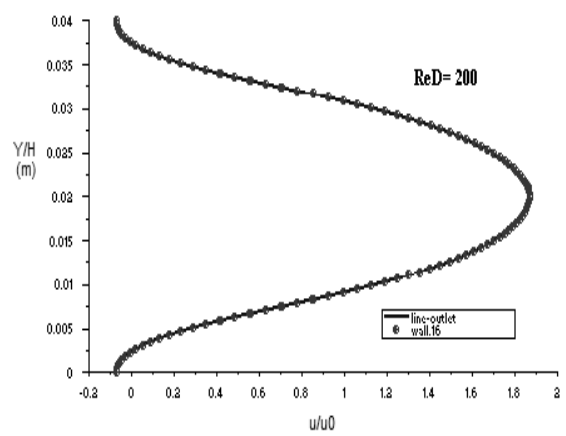

(a)

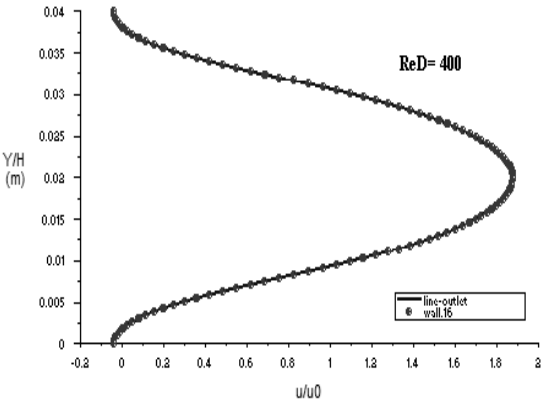

(b)

Figure 7: The developing velocity profiles along the transverse direction of the module outlet and the inlet wall for an in-line arrangement: a $(\operatorname{Re}=200)$ and $b(\operatorname{Re}=400)$.

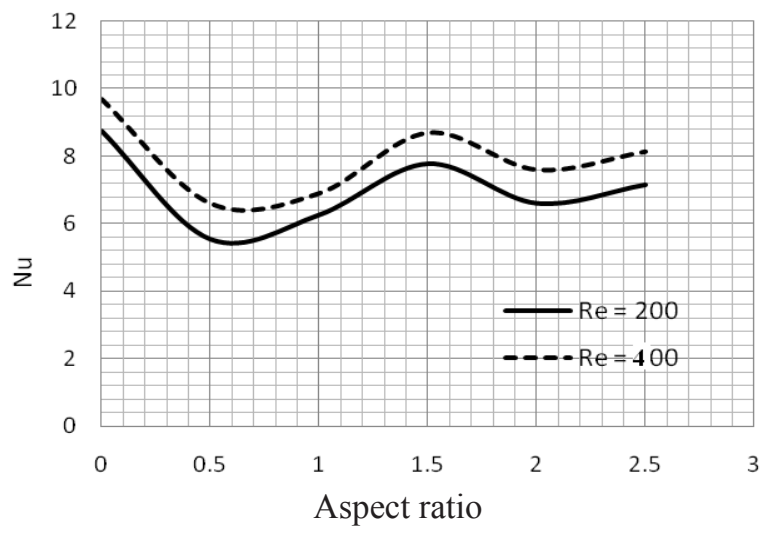

Figure 8: Effect of length aspect ratio on the average Nusselt number for the present module, $\operatorname{Pr}=0.71$, for in-line arrangements for $\mathrm{L} / \mathrm{D}=2.5$ for $\operatorname{Re}=200$ and $\operatorname{Re}=400$.

Figure 9 provides the normalized pressure drop for the present module for inline configurations for $\mathrm{Re}=200$ and $\mathrm{Re}=400$.

It shows the impact of length ratio on the normalized pressure drop. It can be clearly stated that the length ratio has very little effect on pressure drop except for $\mathrm{L} / \mathrm{D}=0$. 


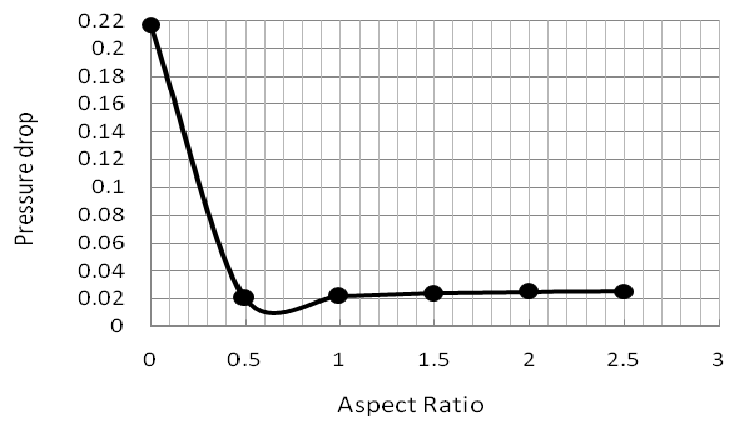

Figure 9: Normalized pressure drop across the present module with aspect ratio for in-line arrangements.

Figure 10 shows comparison between the empirical correlation Martin [9] and the present CFD predictions of in-line circular tube for the variation of $\mathrm{Nu}$ with $\mathrm{Re}_{\mathrm{D}}$ for $\mathrm{L} / \mathrm{D}=2.5$. Good agreement is observed between the present results of the empirical correlations.

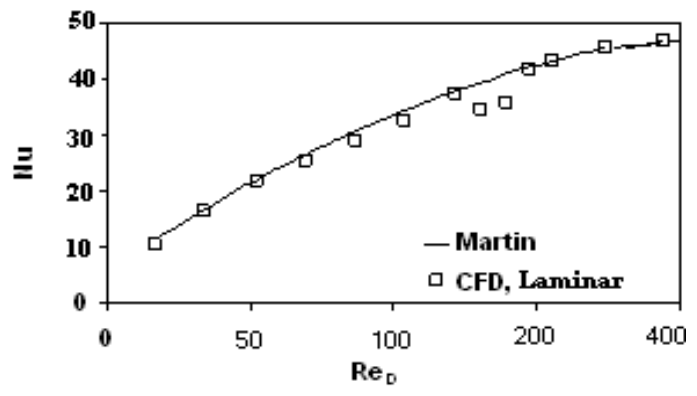

Figure 10: Comparison between the empirical and the present numerical predictions of in-line circular tube for the variation of $\mathrm{Nu}$ with $\mathrm{Re}_{\mathrm{D}}$ for $\mathrm{L} / \mathrm{D}=2.5$.

For validation of the CFD code, fig. 11 shows a comparison between CFD predictions and reference measurements (Iwaki et al. [6]) for the velocity vector as fluid flow (laminar flow) over single circle in cross flow of the selected values of Reynolds numbers $\mathrm{Re}_{\mathrm{D}}$. A reasonable agreement is observed. The results of the present study provides sufficient confidence in using empirical correlations to undertake initial sizing of tube bank heat exchanger design, with thermo-fluid performance subsequently predicted by CFD analysis for specific requirements applications. 


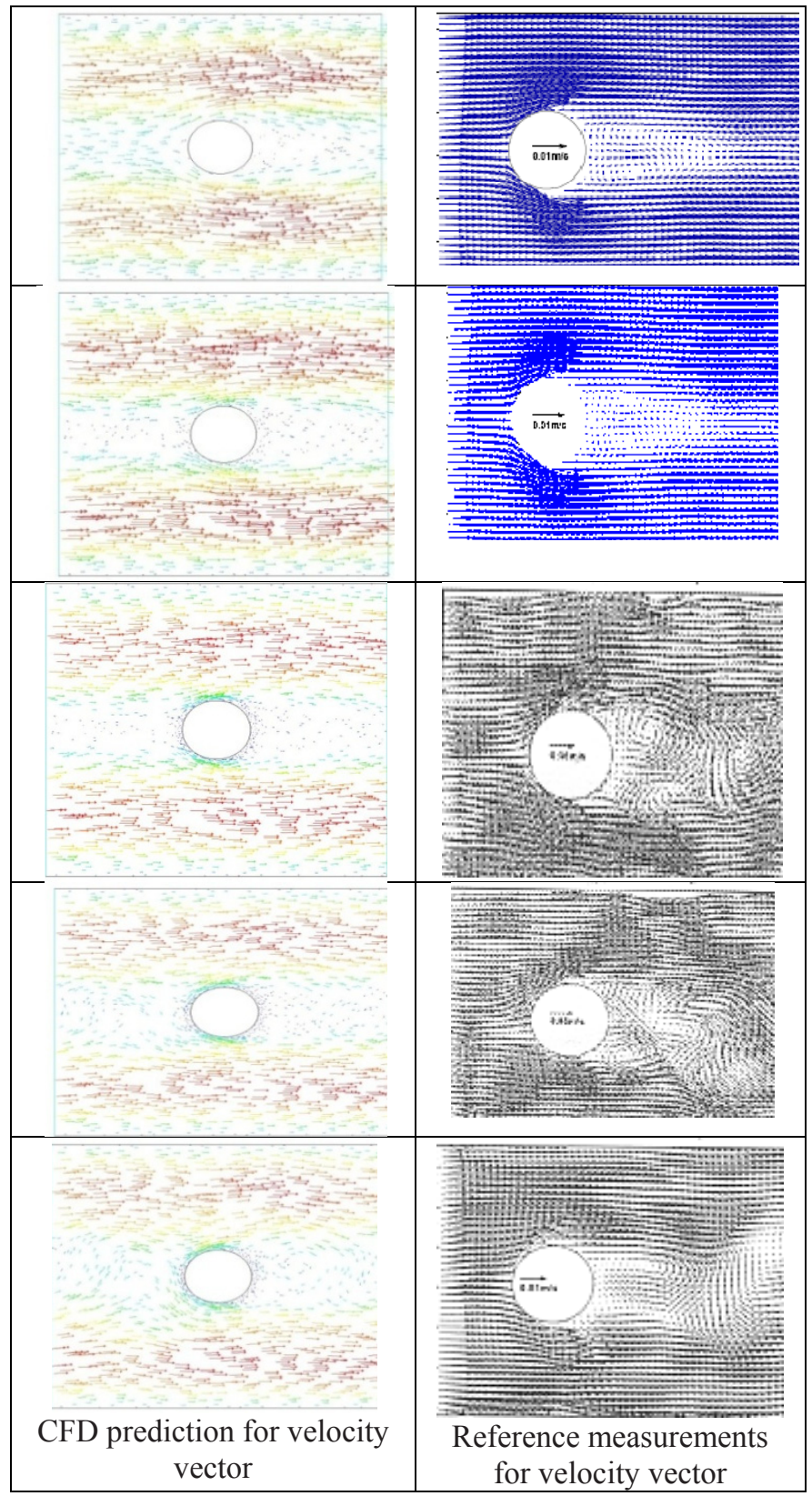

Figure 11: Comparison between present CFD predictions and reference measurements (Iwaki et al. [6]) for the velocity vector as fluid flow over a cylinder in cross flow for the selected values of Reynolds numbers $\mathrm{Re}_{\mathrm{D}}$. 


\section{Conclusions}

1. On comparing CFD predictions with empirical correlations for tube bank convective heat transfer and pressure drop for Re range 50 to 400, good agreement is found with the empirical correlations developed by Martin [9] for heat transfer. Such agreement provides sufficient confidence in using empirical correlations to undertake initial sizing of tube bank heat exchanger design, with thermo fluid performance subsequently by CFD analysis for specific requirements applications.

2. A small change in the heat transfer rate can be predicted since the fluid has almost the same behavior when the length ratio is increased.

3. The length ratio was found to have very little effect on pressure.

4. The maximum velocity in the middle of $(Y / H)$ position as the passage considerably decreases because of a larger flow area.

\section{References}

[1] Webb, R.L., Principles of enhanced heat transfer, John Wiley and Sons, Inc.: New York, 1993.

[2] Versteeg, H.K., Malalasekera, W., An introduction to computational fluid dynamics the finite volume method, Longman Scientific and Technical: New York, 1995.

[3] Hoffman, J.D., Numerical methods for engineers and Scientists, McGrawHill, Inc.: New York, 1992.

[4] Zdrakovich, M.M., Flow around circular cylinders, 1: Fundamental, Oxford University Press: New York, 1997.

[5] Fujii, M., and Fujii, T., A Numerical Analysis of Laminar Flow and Heat Transfer of Air in an In-Line Tube Bank. Numerical Heat Transfer, (7), pp. 89-102, 1984

[6] Iwaki, C., Cheong, K.H., Monji, H., and Matsui, G., PIV Measurement of Vertical Cross Flow Structure over Tube Bundles, Experiments in Fluids, (37), pp. 350-363, 2004.

[7] Karki, K., A calculation procedure for viscous flows at all speeds in complex geometries, Ph. D. Dissertation, University of Minnesota, 1986.

[8] Haitham M. S. Bahaidarah, A Numerical study of heat and momentum transfer over flat tubes, PhD Thesis, Texas A\&M University, August 2004.

[9] Martin, H., The Generalized Lévêque Equation and its Practical Use for the Prediction of Heat and Mass Transfer Rates From Pressure Drop, Chemical Energy, SCI., (57), pp. 3217-3223, 2002.

[10] Hilpert, R., Warmeabgabe Von geheizten Drahten und Rohren, Forsch. Geb. Ingenieurwes, (4), pp. 215-224, 1933.

[11] Zhukauskas, A., Heat Transfer from Tubes in Cross Flow, Advances in Heat Transfer, (8), pp.93-160, 1972. 